\title{
Different Pathology of Peripheral Nerve Sheath Tumor in Neurofibromatosis Type 1: 3 Cases
}

\author{
Gi Jeong Park, Sang Hoon Kim, Ki Hong Kim, Dae Hyun Kim \\ Department of Neurosurgery, Daegu Catholic University Hospital, Catholic University College of Medicine, Daegu, Republic of Korea \\ Corresponding author: \\ Dae Hyun Kim \\ Department of Neurosurgery, Daegu Catholic University \\ Hospital, Catholic University College of Medicine, 33 \\ Duryugongwon-ro 17-gil, Nam-gu, Daegu 42472, \\ Republic of Korea \\ Tel: +82-53-650-3000 \\ Fax: $+82-53-623-7507$ \\ E-mail: daehkim63@gmail.com

\begin{abstract}
Neurofibromatosis type 1 (NF1) is an autosomal-dominant genetic disease that predisposes affected individuals to tumors. Neurofibroma and malignant peripheral nerve sheath tumor (MPNST) are examples of PNSTs that occur either sporadically or as part of hereditary neurocutaneous diseases such as NF1. We treated three patients with NF1 who presented with different PNSTs. All patients underwent surgical resection, and pathologic findings indicated neurofibroma, atypical neurofibroma, and MPNST, respectively. We managed each case based on its pathology. The patient with MPNST died after chemoradiotherapy; the other patients did not experience recurrence for several months.
\end{abstract}

Received: July 20, 2021

Revised: August 13, 2021

Accepted: August 16, 2021
Key Words: Nerve sheath neoplasms; Neurofibroma; Neurofibromatosis 1

\section{INTRODUCTION}

Neurofibromatosis type 1 (NF1) is an autosomal-dominant hereditary disease with a prevalence of about 1 in 3000 . The clinical characteristics of NF1 are café-au-lait spots, neurofibromas, freckling, optic gliomas, and Lisch nodules ${ }^{4,5}$. Neurofibroma is a benign peripheral nerve sheath tumor (PNST) that can transform into atypical neurofibroma, a premalignant tumor, or malignant PNST (MPNST) ${ }^{1,9}$. Because NF1 patients are at higher risk of malignancy, which is the most common cause of death, surgical resection and pathologic confir- mation are very important to the treatment of symptomatic or growing tumors in NF1 patients ${ }^{3,4,11}$. We present three cases of different PNSTs in NF1 patients.

\section{CASE REPORT}

\section{Case 1}

A 23-year-old man with NF1 diagnosed 6 years prior presented with left hip pain. Two round-shaped tumors were found on abdominal computed tomography $(\mathrm{CT})$ and magnetic resonance imaging (MRI). Tumor sizes were $4.2 \times 3.3 \mathrm{~cm}$ and $7.0 \times 4.9 \mathrm{~cm}$ respectively. To reduce left hip pain, we decided to remove these tumors. The patient underwent two separate excisional operations. One originated from the left femoral nerve and the other originated from the left sciatic nerve. Pathologic diagnosis was neurofibroma (Fig. 1). After surgery, hip pain resolved and there were no neurologic complications and no evidence of recurrence on 1-year follow-up CT.

\section{Case 2}

A 25-year-old woman with NF1 diagnosed 7 years prior presented with abdominal pain. On abdominal CT and MRI, a $6.8 \times 4.5-\mathrm{cm}$ sized and oval-shaped mass was found in the right retroperitoneal space with multiple nodular lesions. The size of this mass and the numerous nodular lesions were suspicious for malignancy. For relief of abdominal pain and pathologic confirmation, we performed surgical resection of the mass. The pathologic findings were moderate
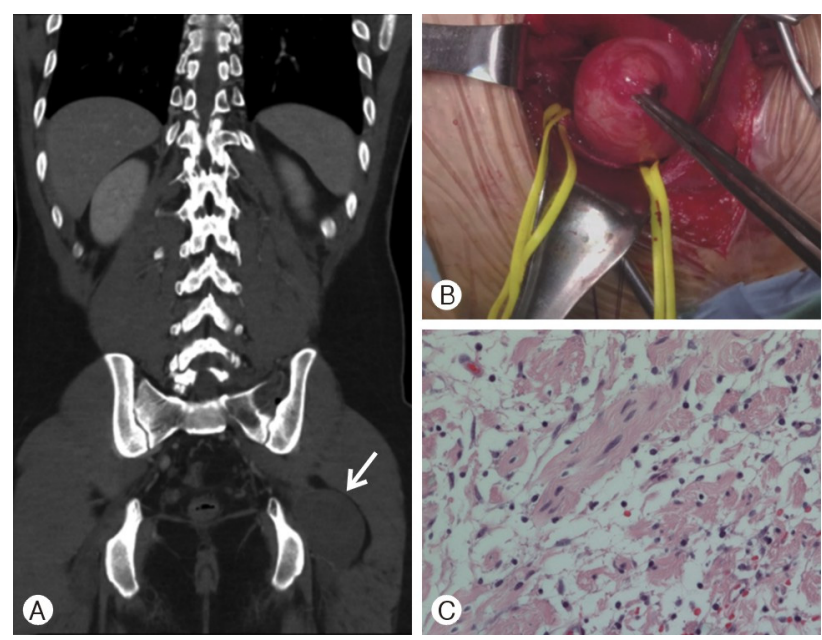

Fig. 1. Case 1. Benign neurofibroma. (A) Computed tomography image of a $7.0 \times 4.9-\mathrm{cm}-\mathrm{sized}$ tumor (arrow). (B) Gross image of excision. (C) Hematoxylin and eosin stain (H\&E), $\times 400$ magnification image of a wary nuclei spindle cells and loose collagenous stroma. 

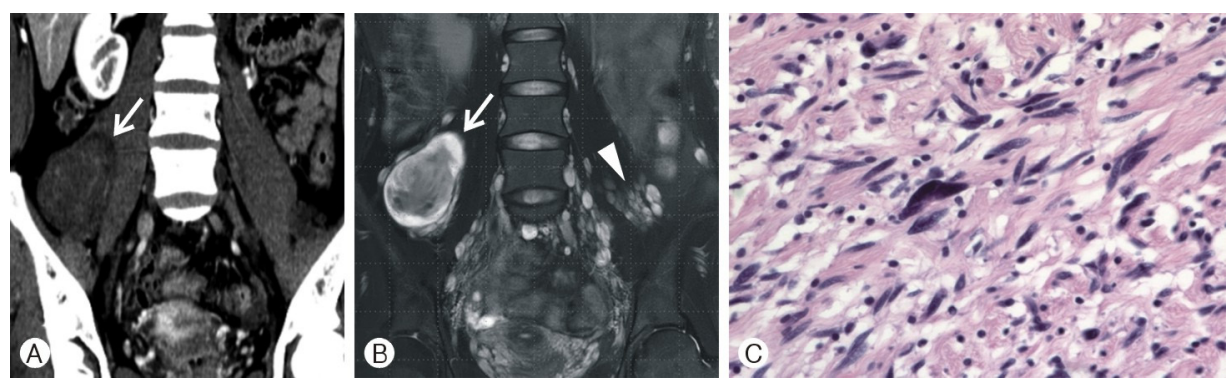

Fig. 2. Case 2. Atypical neurofibroma. (A) Computed tomography of a $6.8 \times 4.5-\mathrm{cm}$-sized tumor (arrow). (B) Fat-saturated T2-weighted magnetic resonance image of a tumor (arrow) without perilesional soft tissue edema. Multiple nodular lesions (arrowhead). (C) Hematoxylin and eosin stain (H\&E), $\times 400$ magnification image of a neurofibroma with focal nuclear atypia and increased cellularity without mitotic activity.
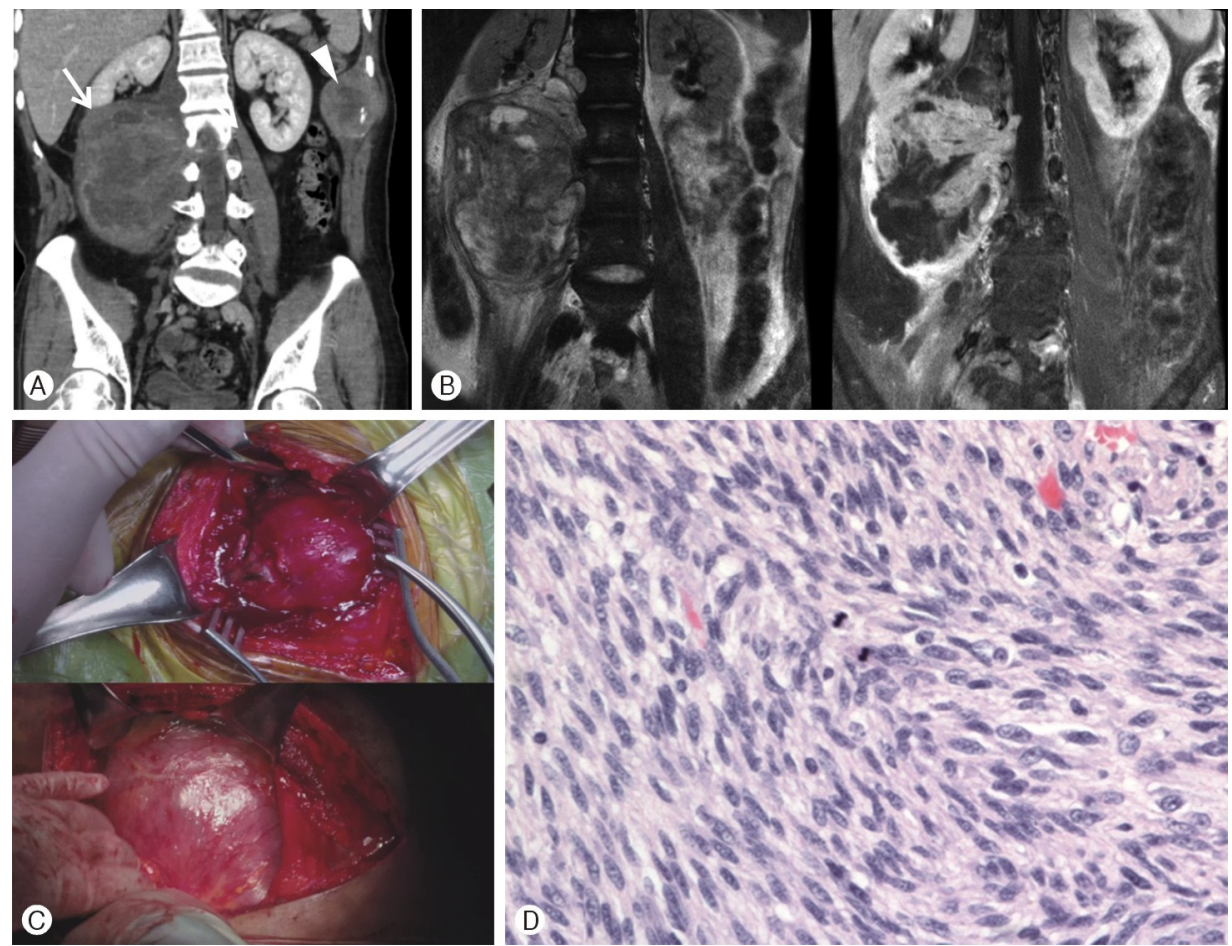

Fig. 3. Case 3. Malignant peripheral nerve sheath tumor. (A) Computed tomography image of a $12.8 \times$ 9.2-cm-sized (arrow) and $5.1 \times 4.1-\mathrm{cm}$-sized (arrowhead) tumor, (B) T2-weighted (left) and enhanced $\mathrm{T} 1$-weighted (right) magnetic resonance image of a tumor with perilesional soft tissue edema, peripheral enhancement pattern and intratumoral cystic lesion. (C) Gross image of excision. (D) Hematoxylin and eosin stain (H\&E), $\times 400$ magnification image of a proliferation of spindle cells with numerous mitoses.

cellularity with nuclear atypia, leading to a diagnosis of atypical neurofibroma (Fig. 2). Abdominal pain was relieved postoperative without complications. On 1-year follow-up CT and MRI, there was no evidence of tumor recurrence or malignant changes.

\section{Case 3}

A 22-year-old man with NF1 diagnosed 6 years prior presented with left flank pain. We found $5.1 \times 4.1-\mathrm{cm}$-sized and $12.8 \times 9.2-\mathrm{cm}$ sized tumors on abdominal CT and MRl. Radiologic findings were large-sized tumors with perilesional edematous changes and peripheral enhancement that are suggestive of malignancy. He underwent two separate excisional operations. These tumors originated from the left intercostal nerve and right lumbar plexus and were pathologically diagnosed as MPNST with cellular atypia and numerous mitoses (Fig. 3). The patient received adjuvant radiotherapy, but follow-up $\mathrm{CT}$ revealed tumor recurrence. The patient underwent reoperation 2 months after the first operation (Fig. 4). Postoperatively, he received chemotherapy, but 3 months later the patient died from tumor progression and complications. 

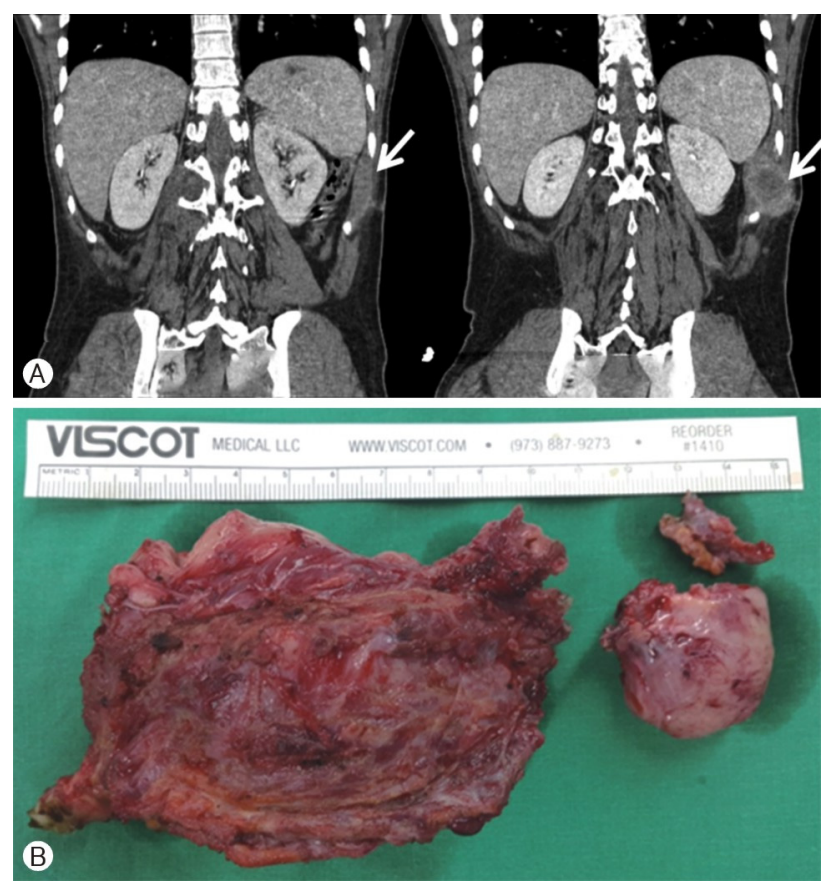

Fig. 4. Case 3. Recurrence of tumor. (A) Postoperative day 30 and 42 computed tomography image of tumor recurrence. (B) Gross image of excised tumor.

\section{DISCUSSION}

MPNST is a rare disease that is very concerning due to its poor prognosis. The incidence of MPNST is approximately $0.001 \%$ in the general population. The recurrence rate is approximately $40 \%$ to $65 \%$, and metastasis is found in $16 \%$ to $39 \%$ of patients. The 5 -year sunvival rate is approximately $25 \%$ to $42 \%{ }^{2,3,7}$. Factors related prognosis include tumor size, the extent of resection in surgery, and rate of mitosis. Total resection or wide tumor excision is considered appropriate because chemotherapy and radiotherapy tend to be ineffective $^{2,7)}$. In case 3, we performed surgical resection, radiotherapy, and chemotherapy, but the patient experienced extremely rapid progression of malignant tumor. Despite potential perioperative complications including neurologic deficits, pathologic confirmation and surgical treatment of MPNST is important.

NFl patients are at high risk of neoplastic disease; the overall risk of cancer in NF1 patients is 2.7 times higher and the risk of malignancy in 50 -year-olds is approximately $20 \%^{11)}$. Also, approximately $50 \%$ of MPNSTs are related to NF1. In NF1 patients, MPNST is less rare than in the general population. The incidence is around $4.6 \%$ and the lifetime risk is $8 \%$ to $13 \%$. Furthermore, the prognosis of MPNST with NF1 is poorer ${ }^{2,3)}$. Therefore, appropriate surveillance is required for $\mathrm{NF1}$ patients. A particularly dose follow-up examination is necessary in cases of growing neurofibroma.

We observed nuclear atypia without mitotic activity on histology in case 2. One-year follow-up CT and MRI revealed no recurrence. However, atypical cell can be found in gray zone between benign and malignant lesions, and transform into malignant tumors in specific genetic characteristics such as deletion of CDKN2A/B. Therefore, atypical neurofibroma is considered a premalignant tumor, ${ }^{1,9)}$. Because of the possibility of malignant change, detection and treatment of atypical neurofibroma are important in NFI patients. Serial MRI and 18F-fluorodeoxyglucose-positron emission tomography can be used for detection of atypical neurofibroma ${ }^{10}$.

Before pathologic confirmation, imaging findings are used for the differentiation of benign and malignant lesions. Differentiation using image findings is helpful for treatment planning. Previous studies described MRI findings indicative of malignancy, including large size, a peripheral enhancement pattern, perilesional soft-tissue edema, and intratumoral cystic lesions ${ }^{8,12)}$. In case 3, these features were observed on MRI before surgery, leading to suspicion of MPNST. Additional radiotherapy was planned. In case 2, there was no peripheral enhancement pattern, perilesional soft-tissue edema, or intratumoral cystic lesions. However, the large tumor size and distinct multiple nodular lesions on MRI suggested a malignant or atypical lesion ${ }^{6)}$. Our experience suggests that MRI findings are important and useful for the differentiation of three types PNSTs.

\section{CONCLUSION}

When dealing with PNSTs in NF1 patients, neurosurgeons should consider the possibility of malignant changes. Further study and specific management plans are needed for PNSTs in NFI.

\section{CONFLICTS OF INTEREST}

No potential conflict of interest relevant to this article was reported.

\section{REFERENCES}

1. Beert E, Brems H, Daniëls B, De Wever I, Van Calenbergh F, Schoenaers J, et al.: Atypical neurofibromas in neurofibromatosis type 1 are premalignant tumors. Genes Chromosomes Cancer 50:1021-1032, 2011

2. Ducatman BS, Scheithauer BW, Piepgras DG, Reiman HM, Ilstrup DM: Malignant peripheral nerve sheath tumors. A clinicopathologic study of 120 cases. Cancer 57:2006-2021, 1986

3. Evans DG, Baser ME, McGaughran J, Sharif S, Howard E, Moran A: Malignant peripheral nerve sheath tumours in neurofibromatosis 1. J Med Genet 39:311-314, 2002

4. Friedman JM: Epidemiology of neurofibromatosis type 1. Am J Med Genet 89:1-6, 1999

5. Friedman JM: Neurofibromatosis 1: clinical manifestations and diagnostic criteria. J Child Neurol 17:548-554; discussion 571572, 646-651, 2002

6. Higham CS, Dombi E, Rogiers A, Bhaumik S, Pans S, Connor SEJ, et al.: The characteristics of 76 atypical neurofibromas as precursors to neurofibromatosis 1 associated malignant peripheral nerve sheath tumors. Neuro Oncol 20:818-825, 2018

7. Hruban RH, Shiu MH, Senie RT, Woodruff JM: Malignant 
peripheral nerve sheath tumors of the buttock and lower extremity. A study of 43 cases. Cancer 66:1253-1265, 1990

8. Li CS, Huang GS, Wu HD, Chen WT, Shih LS, Lii JM, et al.: Differentiation of soft tissue benign and malignant peripheral nerve sheath tumors with magnetic resonance imaging. Clin Imaging 32:121-127, 2008

9. Miettinen MM, Antonescu CR, Fletcher CDM, Kim A, Lazar AJ, Quezado MM, et al.: Histopathologic evaluation of atypical neurofibromatous tumors and their transformation into malignant peripheral nerve sheath tumor in patients with neurofibromatosis 1-a consensus overview. Hum Pathol 67:1-10, 2017

10. Nelson CN, Dombi E, Rosenblum JS, Miettinen MM, Lehky
TJ, Whitcomb PO, et al. Safe marginal resection of atypical neurofibromas in neurofibromatosis type 1 . J Neurosurg [epub ahead of print, 2019. doi: 10.3171/2019.7.Jns191353]

11. Walker L, Thompson D, Easton D, Ponder B, Ponder M, Frayling I, et al.: A prospective study of neurofibromatosis type 1 cancer incidence in the UK. Br J Cancer 95:233-238, 2006

12. Wasa J, Nishida Y, Tsukushi S, Shido Y, Sugiura H, Nakashima $\mathrm{H}$, et al.: MRI features in the differentiation of malignant peripheral nerve sheath tumors and neurofibromas. AJR Am J Roentgenol 194:1568-1574, 2010 